\title{
BMJ Open Effectiveness of $\beta$-blockers in physically active patients with hypertension: protocol of a systematic review
}

\author{
Dagmar Tučková, ${ }^{1,2}$ Miloslav Klugar, ${ }^{1,2}$ Eliška Sovová, ${ }^{3}$ Markéta Sovová, ${ }^{4}$ \\ Lenka Štégnerová ${ }^{3}$
}

To cite: Tučková D,

Klugar M, Sovová E, et al. Effectiveness of $\beta$-blockers in physically active patients with hypertension: protocol of a systematic review. BMJ Open 2016;6: 010534 .

doi:10.1136/bmjopen-2015010534

- Prepublication history and additional material is available. To view please visit the journal (http://dx.doi.org/ 10.1136/bmjopen-2015010534).

Received 12 November 2015 Revised 7 March 2016 Accepted 16 March 2016

CrossMark

For numbered affiliations see end of article.

Correspondence to Dr Miloslav Klugar; miloslav.klugar@upol.cz

\section{ABSTRACT}

Introduction: Based on more than 5 decades of epidemiological studies, it is now widely accepted that higher physical activity patterns and levels of cardiorespiratory fitness are associated with better health outcomes. Therefore, it is necessary to consider how treatment methods affect these two components. Clinically, one very important question concerns the influence of aerobic performance on patients being treated for hypertension. The administration of $\beta$ blockers can significantly reduce maximal-and especially submaximal-aerobic exercise capacity. The objective of this review is to determine, by comparison of existing mono and combination therapy, which $\beta$ blockers are less physically limiting for patients with hypertension who are physically active.

Methods: A three-step strategy will be adopted in the review, following the methods used by the Joanna Briggs Institute (JBI). The initial search will be conducted using the MEDLINE and EMBASE databases. The second search will involve the listed databases for the published literature (MEDLINE, Biomedica Czechoslovaca, Tripdatabase, Pedro, EMBASE, the Cochrane Central Register of Controlled Trials, Cinahl, WoS) and the unpublished literature (Open Grey, Current Controlled Trials, MedNar, ClinicalTrials.gov, Cos Conference Papers Index, the International Clinical Trials Registry Platform of the WHO). Following the JBI methodology, analysis of title/ abstracts and full texts, critical appraisal and data extraction will be carried out on selected studies using the JBI tool, MAStARI. This will be performed by two independent reviewers. If possible, statistical metaanalysis will be pooled. Statistical heterogeneity will be assessed. Subgroup analysis will be used for different age and gender characteristics. Funnel plots, Begg's rank correlation and Egger's regression test will be used to detect or correct publication bias.

Ethics and dissemination: The results will be disseminated by publishing in a peer-reviewed journal. Ethical assessment is not needed-we will search/ evaluate the existing sources of literature.

Trial registration number: CRD42015026914.

\section{BACKGROUND}

High-blood pressure (BP) is one of the most important risk factors in the development of cardiovascular diseases. ${ }^{1}$ In 2013, the European Society of Cardiology and the European Society of Hypertension set out new guidelines for the management of arterial hypertension.

Appropriate lifestyle changes are the cornerstone for the prevention and cure of hypertension. The recommended lifestyle measures that have been shown to be effective in reducing $\mathrm{BP}$ are salt restriction, moderation of alcohol consumption, change of diet, weight reduction and regular physical activity such as moderate aerobic exercise 5-7 days per week. ${ }^{2-4}$

The second part of the therapy is pharmacological. Current guidelines reconfirm that diuretics, $\beta$-blockers, calcium antagonists, ACE inhibitors and angiotensin receptor blockers are all suitable for the initiation and maintenance of antihypertensive treatment.

$\beta$-Blockers are among the most commonly used medications in the treatment of hypertension, especially with regard to the development of cardiovascular complications ${ }^{5}$ such as angina, myocardial infarction, various types of arrhythmias, control of atrial fibrillation rate, ${ }^{6}$ chronic heart failure, hyperadrenergic states such as a thyrotoxicosis, migraines, ${ }^{7}$ or as a form of cardioprotection in patients with anthracycline-induced cardiotoxicity. ${ }^{8} \beta$-Blockers can also improve endothelial dysfunction. ${ }^{9}$

$\beta$-Blockers have different pharmacological properties, such as $\beta-1$ selectivity, intrinsic sympathomimetic activity, and vasodilatory effects with $\alpha$ adrenergic blocking properties and the production of nitric oxide. They may also have hydrophilic and lipophilic properties. This class is in fact a very diverse group of medications with a wide range of properties. $^{5}$

Based on more than five decades of epidemiological studies, it is now widely accepted that higher levels of physical activity and cardiorespiratory fitness are associated with better health outcomes. ${ }^{10}$ Clinically, one very 
important question concerns how the treatment of hypertension influences aerobic performance. ${ }^{2}$ The administration of $\beta$-blockers can significantly reduce maximal, and especially submaximal, aerobic exercise capacity. ${ }^{11}$ Impaired chronotropic response to exercise stress testing is a predictor of mortality. ${ }^{12} \beta$-Blockers can cause a reduction in resting metabolic rate. ${ }^{13}$ Both findings raise the question as to whether treating hypertension using $\beta$-blockers is always appropriate, and which drug, in which form, least affects cardiorespiratory fitness.

Many trials have evaluated the effects of $\beta$-blockers in patients with hypertension, with the endpoints being allcause mortality, morbidity and cardiovascular events; 514 however, few studies have evaluated the influence of $\beta$-blocker therapy on patients' cardiorespiratory fitness and exercise capacity. Billeh et $a l^{15}$ studied the effect of administering $50 \mathrm{mg}$ metoprolol versus $25 \mathrm{mg}$ carvedilol to 12 healthy participants. The $\mathrm{O}_{2}$ peak consumption was significantly reduced by metoprolol but not by carvedilol. Koshucharova $e t a l^{16}$ compared the effect of carvedilol and bisoprolol on healthy participants but found no statistically significant difference in the influence on heart rate during exercise. Herman et $a l^{17}$ investigated the different effects of carvedilol and atenolol on plasma norepinephrine during exercise in a group of 12 healthy volunteers, and found that carvedilol blunted the increase in plasma norepinephrine. Nebivolol is a third-generation $\beta$-blocker with vasodilator properties. ${ }^{18}$ Van Bortel and van Baak, ${ }^{19}$ in another study, compared exercise tolerance in healthy volunteers administered with nebivolol $5 \mathrm{mg}$ versus atenolol $100 \mathrm{mg}$ daily; both drugs reduced blood pressure to a similar degree, although atenolol reduced peak exercise heart rate more than nebivolol. Atenolol also reduced peak exercise and endurance, whereas nebivolol was not associated with any change in peak exercise, endurance, or perceived exercise effort. ${ }^{19}$

When comparing different $\beta$-blockers and their influence on patients with cardiovascular disease, different effects were found. ${ }^{20}$ Marazzi $e t a l^{21}$ compared the effect of nebivolol and carvedilol in hypertensive heart failure patients and found no difference between these two drugs. Metra $e t a l^{20}$ conducted a prospective randomised double-blind comparison of metoprolol and carvedilol, and found that metoprolol led to a greater increase in maximal exercise capacity. Nodari et $a l^{22}$ compared the effect of atenolol versus nebivolol in a group of patients with diastolic heart failure and arterial hypertension. Nebivolol was associated with greater haemodynamic improvement than atenolol.

Exercise testing is used widely for the detection of coronary artery disease, prediction of cardiovascular events, evaluation of physical capacity and effort tolerance, evaluation of exercise-related symptoms, assessment of chronotropic competence and arrhythmias, response to implanted device therapy and assessment of the response to medical interventions. ${ }^{23}$ Current clinical exercise testing procedures involve a predominant dynamic aerobic component. Tread mill and cycle ergometers are the most commonly used dynamic exercise testing devices. Ventilatory expired gas analysis allows the measurement of minute ventilation, $\mathrm{VO}_{2}$ and $\mathrm{VCO}_{2}$, and the combination with ergometers is commonly known as cardiopulmonary exercise testing. The $6 \mathrm{~min}$ walk test is a functional test that can be used to evaluate submaximal exercise capacity. This assessment is frequently used in patients with chronic disease, such as heart failure or chronic obstructive pulmonary disease. ${ }^{24}$

$\mathrm{VO}_{2}$ max is the peak oxygen uptake achieved during exercise performance and is considered the best measure of cardiovascular fitness and exercise capacity. ${ }^{25}$ Exercise capacity is the most powerful predictor of survival. ${ }^{23}$

This systematic review with its extensive search strategy may clarify this issue and influence practice by informing recommendations aimed at physicians and patients with hypertension who want to be physically active.

The preliminary search was conducted using MEDLINE, Prospero, and the JBI Library and Cochrane databases, to establish whether any systematic reviews on this topic had been conducted. The search was performed in October 2015. Neither systematic reviews nor guidelines related to this issue were found.

\section{OBJECTIVE}

The objective of this review is to determine, by comparison of existing mono and combination therapy, which $\beta$-blockers are less physically limiting for patients with hypertension who are physically active.

\section{METHODS}

The protocol was developed according to the Preferred Reporting Items for Systematic Reviews and Meta-Analysis Protocols (PRISMA-P). ${ }^{26}$ This protocol is registered with the PROSPERO prospective register of systematic reviews: CRD42015026914.

\section{STUDY ELIGIBILITY}

\section{Types of participants}

This review will consider studies that include the adult population (both genders, 18 years and older) with any type of hypertension.

\section{Types of interventions}

This review will consider studies that evaluate pharmacological treatment using a $\beta$-blocker (monotherapy or combination therapy); for example, propranolol, metipranolol, sotalol, pindolol, bopindolol, bisoprolol, betaxolol, atenolol, nebovolol, talinolol, esmolol, acebutol, celiprolol, metoprolol, nadolol, $\alpha$ carvedilol.

\section{Types of comparison}

This review will consider studies that evaluate pharmacological treatment using another $\beta$-blocker (monotherapy or combination therapy); for example, propranolol, 
metipranolol, sotalol, pindolol, bopindolol, bisoprolol, betaxolol, atenolol, nebovolol, talinolol, esmolol, acebutol, celiprolol, metoprolol, nadolol, $\alpha$ carvedilol.

\section{Types of outcomes}

This review will consider studies that include the following outcome measures: pharmacological treatment by $\beta$-blockers with the most positive influence on physical activity performance as measured by a maximal or submaximal exercise stress test, or by both a maximal and submaximal test.

The review is primarily focused on patients with hypertension. However, hypertension is often followed by other comorbidities. Other comorbidities with hypertension will be evaluated by a subgroup analysis according to the type and severity of the comorbidity.

\section{Types of studies}

This review will consider primarily experimental study designs, including parallel and crossover RCTs and quasi-experimental studies. If non-experimental study designs are found, the review will also consider epidemiological study designs for inclusion, including prospective and retrospective cohort studies, case-control studies and analytical cross-sectional studies.

\section{SEARCH STRATEGY}

A search strategy will be developed using medical subject headings (eg, MeSH for MEDLINE) and adopted for each database included in the review. Text words related to the issue will also be identified. The search strategy aims to find both, published and unpublished studies. A three-step search strategy will be utilised in this review. An initial limited search of MEDLINE and EMBASE will be undertaken followed by analysis of the text words contained in the title and abstract, and also of the index terms used to describe an article. A second search using all identified keywords and index terms will then be undertaken across all included databases. As a third step, the reference list of all identified reports and articles will be searched for additional studies. Studies published in all possible languages, if they have a title and abstract in English, will be considered for inclusion in this review. Studies published with no time restriction will also be considered for inclusion in this review.

The databases to be searched include:

MedLine@Ovid MEDRLINE(R), Biomedica Czechoslovaca, Tripdatabase, Pedro, EMBASE, Cochrane Central Register of Controlled Trials, Cinahl and Web of Science. The search for unpublished studies will include:

Open Grey, Current Controlled Trials, MedNar, ClinicalTrials.gov, Cos Conference Papers Index and the International Clinical Trials Registry Platform of the WHO.

Search strategy (MEDLINE-Ovid interface):

1. adult* OR adult patient* OR adult population

2. hypertension OR high blood pressure
3. $\beta$ blocker* OR $\beta$-adrenergic blocking agent* OR $\beta$-adrenergic antagonists OR propranolol OR metipranolol OR nadolol OR sotalol OR pindolol OR bopindolol OR betaxolol OR atenolol OR metoprolol OR bisoprolol OR nebovolol OR talinolol OR esmolol OR acebutolol OR celiprolol OR $\alpha$ carvedilol

4. physical activity* OR physical exercise OR physical movement

5. maximal stress test OR cardiac stress test $\mathrm{OR} \mathrm{VO}_{2}$ max test OR submaximal stress test.

The search strategy for the MEDLINE-EMBASE interface is attached in online supplementary appendix I.

\section{STUDY RECORDS}

The literature search results will be uploaded to EndNote X7, and shared by all authors of the review. This will enable a collaboration among reviewers during the process of study selection. Two reviewers (DT and MK) will independently screen and select studies for possible inclusion in the study in two phases. In the first phase, titles and abstracts will be analysed. In the second phase, all possible relevant full texts will be analysed. Any disagreements will be resolved by discussion and a third reviewer (ES).

\section{RISK OF BIAS IN INDIVIDUAL STUDIES}

Papers selected for retrieval will be assessed by two independent reviewers (DT and MK) for methodological quality prior to inclusion in the review, using standardised critical appraisal instruments from the JBI Meta Analysis of Statistics Assessment and Review Instrument (JBI-MAStARI) (see online supplementary appendix II). ${ }^{27}$ Any disagreements that arise between the reviewers will be resolved by discussion and a third reviewer (ES).

\section{DATA COLLECTION PROCESS}

Data will be extracted independently by reviewers (DT and MK) from papers included in the review, using the standardised data extraction tools from JBI-MAStARI for RCTs and pseudo-randomised trials, for prospective and retrospective cohort studies, for case-control studies and for cross-sectional studies (see online supplementary appendix III) ${ }^{27}$ The data extracted will include specific details about the interventions, populations, study methods and outcomes of significance, of the review objectives. Any disagreement will be resolved by discussion.

\section{DATA ITEMS/DEALING WITH MISSING DATA}

We will extract the generic and trade names of the interventions and compare them in terms of use control, dosage, frequency and duration of treatment, pharmacological drugs and patient characteristics (age, gender, given disease, physical activity and type of hypertension). Whenever possible, we will use the results from an intention-to-treat analysis. If effect size cannot be 
calculated, we will contact the authors for additional data. The authors of the included studies will be contacted, when necessary, to gather relevant information.

\section{OUTCOMES AND PRIORITISATION}

The primary outcome will be to establish which $\beta$-blockers are the most effective in physically active patients with hypertension, in terms of minimal limitation of physical activity performance. Data relating to the following questions will be sought:

1 . Which $\beta$-blockers enable the patients with hypertension to be physically active with no performance limitation?

2. Which $\beta$-blockers help patients with hypertension to be physically active and are most effective in treating hypertension?

3. Which $\beta$-blockers help patients with hypertension and comorbidities to be physically active, and which are most effective in treating hypertension?

\section{DATA SYNTHESIS}

Quantitative data will, where possible, be pooled in statistical meta-analyses, using JBI-MAStARI. All results will be subject to double-data entry. Effect sizes expressed as OR (for categorical data) and weighted mean differences (for continuous data) and their 95\% CIs will be calculated for analysis. If we retrieve homogeneous RCTs, we will not include any other study design, and we will then perform fixed-effects meta-analyses to synthesise the data by pooling the results of included studies. If we do not retrieve homogeneous RCTs, or have to include other study designs, we will perform random-effects meta-analyses. We will pool studies with similar designs, for example, the data from RCTs will not be pooled with data from quasi-randomised trials or non-randomised trials. Where statistical pooling is not possible, the findings will be presented in narrative form, including tables and figures to aid in data presentation where appropriate.

\section{ASSESSIMENT OF HETEROGENEITY}

Initially, clinical heterogeneity will be assessed by determining whether the studies are sufficiently similar to pool in terms of inclusion criteria. If they are clinically homogeneous, statistical heterogeneity will be assessed using the standard $\chi^{2}$ test (significance level: 0.1 ) and $\mathrm{I}^{2}$ statistic, with a value of $\mathrm{I}^{2} \geq 50 \%$ indicating significant heterogeneity. If statistical heterogeneity is found, it will be assessed by sensitivity and subgroup analyses.

\section{SENSITIVITY ANALYSIS}

To ensure sensitivity analysis, we will exclude all studies that are appraised as having a high risk of bias. The sensitivity analysis will be used to assess the robustness of the results to specific decisions made and methods used.

\section{SUBGROUP ANALYSIS}

Subgroup analysis will be used for different age and gender characteristics. Another subgroup analysis will be used for a different level of physical activity, as measured by $\mathrm{VO}_{2}$ max. Subgroup analysis will be used for different stress tests. The next subgroup analysis will be used for different levels of drug dosage. Subgroup analysis will also be used for hypertension and different comorbidities, according to their type and severity. $\beta$-blockers have different pharmacodynamics and pharmacokinetic properties, so subgroup analysis will be used for hydrophilicity, lipophilicity, intrinsic sympathomimetic activity and vasodilatory properties. We also will pay attention to another subgroup due to the category of the nature of $\beta$-blockers, which are divided into non-selective, $\beta-1$ selective, $\alpha$ and $\beta$-blocker, and partial antagonist.

\section{META-BIAS ASSESSMENT}

We plan to provide assessment of publication bias based on whether the RCT protocols were published before the research on the patients or respondents was conducted-for published as well as unpublished studies (see 'Search strategy'). The fixed-effect estimate versus the random-effect model will be evaluated to allow the presence of bias in the published sources of literature to be assessed. For reporting the potential reporting bias, we will use funnel plots if $\geq 10$ studies are available. We will use Begg's rank correlation and Egger's regression tests for detecting or correcting publication bias.

\section{CONFIDENCE IN CUMULATIVE EVIDENCE}

Based on the results and quality of evidence, the tool known as 'Grading of Recommendation Assessment, Development and Evaluation' (GRADE) ${ }^{28}$ will be used. The quality of evidence will be assessed across the domains of risk of bias, consistency, directness, precision and publication bias. Quality will be assessed as high (further research is very unlikely to change our confidence in the estimate of effect) or moderate (further research is likely to have an important impact on our confidence in the estimate of effect and may change the estimate) or low (further research is very likely to have an impact on our confidence in the estimate of effect and is likely to change the estimate) or very low (very uncertain about the estimate of effect).

\section{ETHICS AND DISSEMINATION}

This protocol of a systematic review was created in October 2015. Next, the systematic review development team will start to work on a planned systematic review. The results will be disseminated-focusing on patients and experts in cardiovascular practice-by publication in a peer-reviewed journal. Ethical assessment is not needed because we will search and evaluate only existing sources of literature. 
Author affiliations

${ }^{1}$ Department of Social Medicine and Public Health, Faculty of Medicine and Dentistry, Palacký University in Olomouc, Olomouc, Czech Republic

${ }^{2}$ The Czech Republic (Middle European) Centre for Evidence-Based Health Care: An affiliated Centre of the Joanna Briggs Institute, Department of Social Medicine and Public Health, Faculty of Medicine and Dentistry, Palacký University in Olomouc, Olomouc, Czech Republic

${ }^{3}$ Department of Exercise Medicine and Cardiovascular Rehabilitation, Faculty of Medicine and Dentistry, Palacký University in Olomouc, Olomouc, Czech Republic

${ }^{4}$ Department of Internal Medicine II - Gastroenterology and Hepatology, Faculty of Medicine and Dentistry, Palacký University in Olomouc, Olomouc, Czech Republic

Acknowledgements The authors would like to thank the Charlesworth Group, for professional editing. This work was supported by the project, 'Hypertension as one of the risk factors in the development of cardiovascular disease', IGA_LF_2015_024, Faculty of Medicine and Dentistry, Palacký University in Olomouc, Czech Republic.

Contributors DT and MK were responsible for the study conception and design. All the authors contributed to the development of the selection criteria, risk of bias assessment strategy and data extraction. MK and DT are methodologists. ES is the content expert for hypertension. MK is the content expert for physical activity. DT, MK, ES, MS, LS read, provided feedback and approved the final manuscript.

Funding This paper was supported by a grant provided by the Faculty of Medicine and Dentistry, Palacký University in Olomouc, Czech Republic, for the project 'Hypertension as one of the major risk factors for developing cardiovascular disease' (IGA_LF_2015_024).

\section{Competing interests None declared.}

Provenance and peer review Not commissioned: externally peer reviewed.

Open Access This is an Open Access article distributed in accordance with the Creative Commons Attribution Non Commercial (CC BY-NC 4.0) license, which permits others to distribute, remix, adapt, build upon this work noncommercially, and license their derivative works on different terms, provided the original work is properly cited and the use is non-commercial. See: http:// creativecommons.org/licenses/by-nc/4.0/

\section{REFERENCES}

1. Perk J, De Backer G, Gohlke $\mathrm{H}$, et al. European Guidelines on cardiovascular disease prevention in clinical practice (version 2012). The Fifth Joint Task Force of the European Society of Cardiology and Other Societies on Cardiovascular Disease Prevention in Clinical Practice (constituted by representatives of nine societies and by invited experts). Eur Heart $J$ 2012;33:1635-701.

2. Mancia G, Fagard R, Narkiewicz K, et al., Task Force Members. 2013 ESH/ESC Guidelines for the management of arterial hypertension: the Task Force for the management of arterial hypertension of the European Society of Hypertension (ESH) and of the European Society of Cardiology (ESC). J Hypertens 2013;31:1281-357.

3. Hackam DG, Khan NA, Hemmelgarn BR, et al. The 2010 Canadian Hypertension Education Program recommendations for the management of hypertension: part 2-therapy. Can J Cardiol 2010;26:249-58.

4. Ogedegbe G, Gyamfi J, Plange-Rhule J, et al. Task shifting interventions for cardiovascular risk reduction in low-income and middle-income countries: a systematic review of randomised controlled trials. BMJ Open 2014:4:e005983.

5. Larochelle $P$, Tobe $S W$, Lacourcière $Y$. $\beta$-Blockers in hypertension: studies and meta-analyses over the years. Can J Cardiol 2014;30: S16-22.
6. Kotecha $\mathrm{D}$, Holmes $\mathrm{J}$, Krum $\mathrm{H}$, et al. Efficacy of $\beta$ blockers in patients with heart failure plus atrial fibrillation: an individual-patient data meta-analysis. Lancet 2014;384:2235-43.

7. Jackson JL, Cogbill E, Santana-Davila R, et al. A comparative effectiveness meta-analysis of drugs for the prophylaxis of migraine headache. PLOS ONE 2015;10:e0130733.

8. Yun S, Vincelette ND, Abraham I. Cardioprotective role of $\beta$-blockers and angiotensin antagonists in early-onset anthracyclines-induced cardiotoxicity in adult patients: a systematic review and meta-analysis. Postgrad Med J 2015;91:627-33.

9. Peller M, Ozierański K, Balsam P, et al. Influence of beta-blockers on endothelial function: a meta-analysis of randomized controlled trials. Cardiol J 2015;22:708-16.

10. Myers J, McAuley P, Lavie CJ, et al. Physical activity and cardiorespiratory fitness as major markers of cardiovascular risk: their independent and interwoven importance to health status. Prog Cardiovasc Dis 2015;57:306-14.

11. Van Baak MA. Beta-adrenoceptor blockade and exercise. An update. Sports Med 1988;5:209-25.

12. Lauer MS, Francis GS, Okin PM, et al. Impaired chronotropic response to exercise stress testing as a predictor of mortality. JAMA 1999;281:524-9.

13. Lamont LS, Brown T, Riebe D, et al. The major components of human energy balance during chronic beta-adrenergic blockade. $J$ Cardiopulm Rehabil 2000;20:247-50.

14. Kuyper LM, Khan NA. Atenolol vs nonatenolol $\beta$-blockers for the treatment of hypertension: a meta-analysis. Can J Cardiol 2014;30: S47-53.

15. Billeh R, Hirsh D, Barker C, et al. Randomized, double-blind comparison of acute beta1-blockade with $50 \mathrm{mg}$ metoprolol tartrate vs $25 \mathrm{mg}$ carvedilol in normal subjects. Congest Heart Fail 2006;12:254-7.

16. Koshucharova G, Klein W, Lercher $P$, et al. Different beta-blocking effects of carvedilol and bisoprolol in humans. J Clin Basic Cardiol 2001;4:53-6.

17. Herman RB, Jesudason PJ, Mustafa AM, et al. Differential effects of carvedilol and atenolol on plasma noradrenaline during exercise in humans. Br J Clin Pharmacol 2003;55:134-8.

18. Howlett JG. Nebivolol: vasodilator properties and evidence for relevance in treatment of cardiovascular disease. Can J Cardiol 2014;30:S29-37.

19. Van Bortel LM, van Baak MA. Exercise tolerance with nebivolol and atenolol. Cardiovasc Drugs Ther 1992;6:239-47.

20. Metra M, Giubbini R, Nodari S, et al. Differential effects of beta-blockers in patients with heart failure: a prospective, randomized, double-blind comparison of the long-term effects of metoprolol versus carvedilol. Circulation 2000;102:546-51.

21. Marazzi G, Volterrani M, Caminiti G, et al. Comparative long term effects of nebivolol and carvedilol in hypertensive heart failure patients. J Card Fail 2011;17:703-9.

22. Nodari S, Metra M, Dei Cas L. Beta-Blocker treatment of patients with diastolic heart failure and arterial hypertension. A prospective, randomized, comparison of the long-term effects of atenolol vs. nebivolol. Eur J Heart Fail 2003;5:621-7.

23. Fletcher GF, Ades PA, Kligfield P, et al. Exercise standards for testing and training a scientific statement from the American Heart Association. Circulation 2013;128:873-934.

24. Laboratories ACoPSfCPF. ATS statement: guidelines for the six-minute walk test. Am J Respir Crit Care Med 2002;166:111.

25. McArdle WD, Katch FI, Katch VL. Exercise physiology: nutrition, energy, and human performance. Lippincott Williams \& Wilkins, 2010.

26. Moher D, Shamseer L, Clarke M, et al. Preferred reporting items for systematic review and meta-analysis protocols (PRISMA-P) 2015 statement. Syst Rev 2015;4:1.

27. The JBI. Joanna Briggs Institute Reviewer's Manual. 2014. University of Adelaide, 2014.

28. Munn Z, Porritt K, Aromataris E, et al. Summary of findings tables for Joanna Briggs Institute systematic reviews. Joanna Briggs Institute, 2014. 